\title{
Asymptotic Numerical Solutions for Second-Order Quasilinear Singularly Perturbed Problems
}

\author{
Chein-Shan Liu \\ National Taiwan Ocean University, Keelung, Taiwan \\ Chih-Wen Chang \\ National United University, Miaoli, Taiwan, cwchang@nuu.edu.tw
}

Follow this and additional works at: https://jmstt.ntou.edu.tw/journal

Part of the Fresh Water Studies Commons, Marine Biology Commons, Ocean Engineering Commons, Oceanography Commons, and the Other Oceanography and Atmospheric Sciences and Meteorology Commons

\section{Recommended Citation}

Liu, Chein-Shan and Chang, Chih-Wen (2022) "Asymptotic Numerical Solutions for Second-Order Quasilinear Singularly Perturbed Problems," Journal of Marine Science and Technology: Vol. 29: Iss. 6, Article 3.

DOI: $10.51400 / 2709-6998.2554$

Available at: https://jmstt.ntou.edu.tw/journal/vol29/iss6/3

This Research Article is brought to you for free and open access by Journal of Marine Science and Technology. It has been accepted for inclusion in Journal of Marine Science and Technology by an authorized editor of Journal of Marine Science and Technology. 


\title{
Asymptotic Numerical Solutions for Second-order Quasilinear Singularly Perturbed Problems
}

\author{
Chein-Shan Liu ${ }^{a}$, Chih-Wen Chang ${ }^{\mathrm{b}, *}$ \\ ${ }^{a}$ Center of Excellence for Ocean Engineering, Center of Excellence for the Oceans, National Taiwan Ocean University, 202, No.2, \\ Beining Rd., Keelung, Taiwan \\ ${ }^{\mathrm{b}}$ Department of Mechanical Engineering, National United University, 360302, No. 2, Lienda Rd., Miaoli, Taiwan
}

\begin{abstract}
For a second-order quasilinear singularly perturbed problem under the Dirichlet boundary conditions, we propose a new asymptotic numerical method, which involves two problems: a reduced problem with a one-side boundary condition and a novel boundary layer correction problem with a two-sided boundary condition. Through the introduction of two new variables, both problems are transformed to a set of three first-order initial value problems with zero initial conditions. The Runge-Kutta method is then applied to integrate the differential equations and to determine two unknown terminal values of the new variables until they converge. The modified asymptotic numerical solution satisfies the Dirichlet boundary conditions. Some examples confirm that the newly proposed method can achieve a better asymptotic solution to the quasilinear singularly perturbed problem. For most values of the perturbing parameter, the present method not only preserves the inherent asymptotic property within the boundary layer but also improves the accuracy within the entire domain.
\end{abstract}

Keywords: Quasilinear singularly perturbed problem, Asymptotic numerical method, Initial value problem method, Modified asymptotic solution

\section{Introduction}

$\mathrm{T}$ he singularly perturbed problem (SPP) involves the second-order derivative term being multiplied by a small parameter whose perturbation operates over a narrow region, across which the solution undergoes a rapid change. The thin layer frequently adjoins the boundaries of a given interval because a small parameter multiplies the highest derivative term in the differential equation. These phenomena are usually referred to as the boundary layer in fluid mechanics, edge layer in solid mechanics, and skin layer in electronics. Standard numerical methods often fail to work for the SPP when the perturbing parameter is sufficiently small. To overcome this difficulty, special numerical methods for the SPP have been developed [1-13].
Various asymptotic approximation methods have been applied to solve the SPP. Numerical methods that are effective have been developed based on asymptotic matching of boundary layer behavior; this is uniformly valid with respect to the perturbing parameter [14]. The resulting asymptotic solution usually only satisfies the boundary condition on the side of the boundary layer, but it does not precisely match the boundary condition on the other side. Therefore, we improve the conventional asymptotic solution by proposing a modified asymptotic approximation. Scholars have solved the SPP by dividing the domain of the problem into nonoverlapping outer and inner regions with a terminal point near the boundary layer [15-18]. Within each region, the governing equation for different types is given with two boundary conditions attached. We

Received 22 June 2021; revised 16 August 2021; accepted 10 September 2021.

Available online 27 December 2021

* Corresponding author.

E-mail address: cwchang@nuu.edu.tw (C-W. Chang). 
provide an example to demonstrate this method and highlight its drawback.

The decomposition method [19-22] has been broadly used to determine an asymptotic expansion of the SPP because of its advantages in asymptotic analysis for resolving two subproblems. We modified the original problem into a reduced problem and a boundary layer correction problem. No discrepancy concerning the ability of the reduced problem to determine the outer solution is known; however, various techniques are available to construct boundary layer correction problems that yield inner solutions. In this paper, we decompose the numerical process into a coupled first-order outer solution to a second-order inner solution. To ensure the second-order inner solution satisfies the derived boundary conditions, we were inspired by previous works [23-27] to develop a novel initial value problem method that guarantees that the boundary conditions are satisfied. Consequently, we must solve three first-order problems with zero initial values given.

We arrange the paper as follows. Some mathematical preliminaries that prescribe the basic ingredients in the asymptotic analysis for a certain sample are given in Section 2. In Section 3, we decompose the SPP to determine an inner solution and an outer solution to the new proposed boundary layer correction problem. We also introduce a coordinate transformation of the independent variable, resulting in the second-order SPP in the new coordinate being less sharp within the boundary layer. In Section 4, we derive two functions to automatically preserve the boundary conditions. The SPP is transformed to initial value problems (IVPs) for two new variables. An iterative algorithm is developed to determine the unknown right-end values of the new variables, and thus, the modified asymptotic solution can be successfully determined with a few iterations. Some numerical examples are solved in Section 5 by using the proposed asymptotic numerical algorithm. Finally, conclusions are drawn in Section 6.

\section{Mathematical preliminaries}

To demonstrate the basic components of the asymptotic analysis, we begin with the following case:

$\varepsilon u^{\prime \prime}(x)+u^{\prime}(x)+u(x)=0$,

$u(0)=\alpha, u(1)=\beta$, where $\varepsilon>0$ is a sufficiently small perturbing parameter. The exact solution is

$u_{e}(x)=\frac{1}{e^{p_{2}}-e^{p_{1}}}\left[\left(\alpha e^{p_{2}}-\beta\right) e^{p_{1} x}+\left(\beta-\alpha e^{p_{1}}\right) e^{p_{2} x}\right]$,

where

$p_{1}=\frac{-1+\sqrt{1-4 \varepsilon}}{2 \varepsilon}, p_{2}=\frac{-1-\sqrt{1-4 \varepsilon}}{2 \varepsilon}$.

The applicable range of $\varepsilon$ is $0<\varepsilon<0.25$.

We briefly sketch the conventional matched asymptotic method for approximating Eqs. (1) and (2). In the range of $x>\mathrm{O}(\varepsilon)$, we consider a reduced equation:

$u_{o}^{\prime}(x)+u_{o}(x)=0, u_{o}(1)=\beta$,

and obtain the outer solution:

$u_{o}(x)=\beta e^{1-x}$.

To seek the inner solution $u_{i}(x)$, we consider a stretched coordinate:

$\zeta:=\frac{x}{\varepsilon}$,

which changes Eq. (1) to

$\frac{d^{2} u_{i}}{d \zeta^{2}}+\frac{d u_{i}}{d \zeta}+\varepsilon u_{i}=0$.

In the inner region, we fix $\zeta$ and let $\varepsilon \rightarrow 0$, i.e., $x \rightarrow 0$, obtaining

$\frac{d^{2} u_{i}}{d \zeta^{2}}+\frac{d u_{i}}{d \zeta}=0$

which is subjected to the left-end boundary condition:

$u_{i}(0)=\alpha$.

Consequently, the inner solution is given by

$u_{i}=c+(\alpha-c) e^{-\zeta}$,

where $c$ is an integration constant, determined by the matching principle [28]:

$u_{i}^{o}:=\lim _{\zeta \rightarrow \infty} u_{i}=\lim _{x \rightarrow 0} u_{o}=: u_{o}^{i}$,

which leads to $c=e \beta$ in view of Eqs. (11) and (6). Hence, the inner solution is given by

$u_{i}=e \beta+(\alpha-e \beta) e^{-\zeta}$.

Finally, a uniform asymptotic solution denoted as $u_{a}(x)$ is obtained through a composition technique: 


$$
\begin{aligned}
u_{a}(x)=u_{o}+u_{i}-u_{o}^{i} & =u_{o}+u_{i}-u_{i}^{o} \\
& =\beta e^{1-x}+e \beta+(\alpha-e \beta) e^{-\zeta}-e \beta \\
& =\beta e^{1-x}+(\alpha-e \beta) e^{-\zeta} \\
& =\beta e^{1-x}+(\alpha-e \beta) e^{-x / \varepsilon} .
\end{aligned}
$$

We can observe that

$u_{a}(0)=\alpha, u_{a}(1)=\beta+(\alpha-e \beta) e^{-1 / \varepsilon}$,

which means that the solution $u_{a}(x)$ does not match the right-end boundary condition in Eq. (2) with an absolute error of $|\alpha-e \beta| e^{-1 / \varepsilon}$. When $\varepsilon$ is small, the error is negligible; however, when $\varepsilon$ is a moderate value, the error cannot be neglected, which may induce a large error within the entire domain of the original asymptotic solution (14).

To improve this method, we propose a new asymptotic method to solve

$u_{o}^{\prime}(x)+u_{o}(x)=0, u_{o}(1)=\beta$

$\varepsilon u_{i}^{\prime \prime}(x)+u_{i}^{\prime}(x)=0, u_{i}(0)=\alpha-u_{o}(0), u_{i}(1)=0$,

and

$u(x)=u_{o}(x)+u_{i}(x)$

represents a new asymptotic solution to Eqs. (1) and (2). Eqs. (16) and (5) are the same, and Eq. (17) is equivalent to Eq. (9) if Eq. (9) is represented in the original coordinate $x$. Instead of considering one left-end condition by using the matching method to determine the integration constant $c$ and then obtaining the composition solution, we directly subject $u_{i}(x)$ to the two boundary conditions in Eq. (17) and employ the direct sum in Eq. (18) to determine the new asymptotic solution.

We can derive

$u(x)=\beta e^{1-x}+\frac{\alpha-e \beta}{1-e^{-1 / \varepsilon}}\left(e^{-x / \varepsilon}-e^{-1 / \varepsilon}\right)$,

which satisfies both boundary conditions in Eq. (2). By deleting the term $e^{-1 / \varepsilon}$, Eq. (19) is reduced to Eq. (14). Because

$e^{-1 / \varepsilon} \rightarrow 0,1-e^{-1 / \varepsilon} \rightarrow 1$, when $\varepsilon \rightarrow 0$,

the solution in Eq. (19) tends to $u_{a}(x)$ in Eq. (14) when $\varepsilon \rightarrow 0$.

Given $\varepsilon=0.245, \alpha=0$, and $\beta=1$, Fig. 1 plots $u_{e}$, $u_{a}$, and the present result $u$ in Eq. (19) with respect to $x$, where the maximum error (ME) of $\left|u_{e}-u_{a}\right|$ is $8.96 \times 10^{-2}$ and the ME of $\left|u_{e}-u\right|$ is $5.02 \times 10^{-2}$. The present $u$ in Eq. (19) is closer to the exact solution

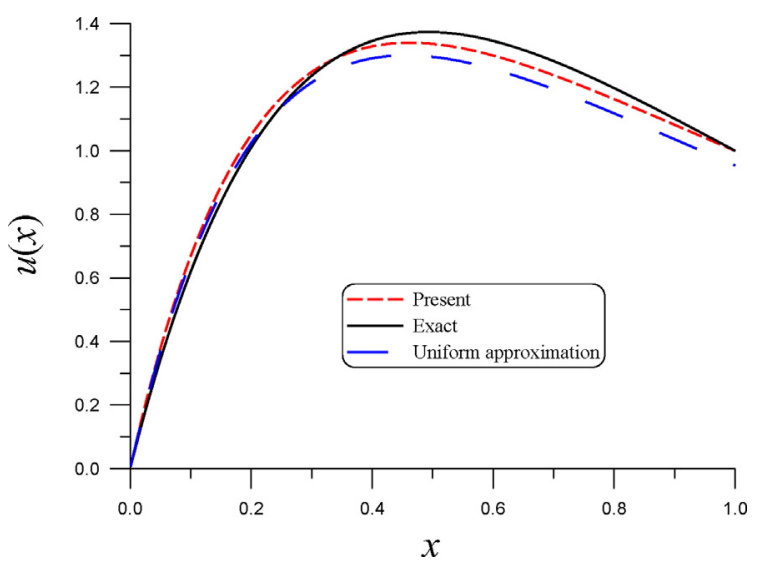

Fig. 1. For a given example, a comparison of the exact solution, uniform approximation, and present solution.

than that of $u_{a}(x)$ in Eq. (14). Table 1 presents a comparison of the ME1 of $\left|u_{e}-u\right|$ and the ME2 of $\left|u_{e}-u_{a}\right|$ for different values of $\varepsilon$, which are the same when $\varepsilon \leq 0.05$. For $\varepsilon \geq 0.1$, ME1 is smaller than ME2. Eqs. (19) and (14) possess the same asymptotic property.

The asymptotic inner boundary condition method proposed by Andargie and Reddy [18] divided the interval into two subintervals divided by a terminal point $x_{p}=\varepsilon \zeta_{p}$, where $\zeta_{p}$ is a parameter to specify the position of the terminal point. For definite, we consider $\alpha=1$ and $\beta=2$ in Eq. (2). In the inner region, we have

$Y^{\prime \prime}(\zeta)+Y^{\prime}(\zeta)=-2 \varepsilon e^{1-\varepsilon \zeta}, 0 \leq \zeta \leq \zeta_{p}$,

$Y(0)=1, Y^{\prime}\left(\zeta_{p}\right)+Y\left(\zeta_{p}\right)=2 e^{1-\varepsilon \zeta_{p}}$,

and in the outer region, we have

$\varepsilon y^{\prime \prime}(x)+y^{\prime}(x)=-2 e^{1-x}, x_{p} \leq x \leq 1$,

$y\left(x_{p}\right)=Y\left(\zeta_{p}\right), y(1)=2$.

For Eqs. (20)-(23), we can derive the exact solutions of $Y(\zeta)$ and $y(x)$ :

Table 1. Comparison of ME1 and ME2 of the present and asymptotic solutions to the exact solution with different $\varepsilon$

\begin{tabular}{lll}
\hline$\varepsilon$ & ME1 & ME2 \\
\hline $\mathbf{0 . 2 4}$ & $4.944 \times 10^{-2}$ & $8.766 \times 10^{-2}$ \\
$\mathbf{0 . 2}$ & $6.402 \times 10^{-2}$ & $8.138 \times 10^{-2}$ \\
$\mathbf{0 . 1}$ & $1.095 \times 10^{-1}$ & $1.097 \times 10^{-1}$ \\
$\mathbf{0 . 0 5}$ & $8.624 \times 10^{-2}$ & $8.624 \times 10^{-2}$ \\
$\mathbf{0 . 0 1}$ & $2.419 \times 10^{-2}$ & $2.419 \times 10^{-2}$ \\
$\mathbf{0 . 0 0 1}$ & $2.674 \times 10^{-3}$ & $2.674 \times 10^{-3}$ \\
$\mathbf{0 . 0 0 0 1}$ & $2.712 \times 10^{-4}$ & $2.712 \times 10^{-4}$ \\
\hline
\end{tabular}


$Y(\zeta)=\frac{2}{1-\varepsilon} e^{1-\varepsilon \zeta}+\left(1-\frac{2 e}{1-\varepsilon}\right) e^{-\zeta}$

$y(x)=\frac{2}{1-\varepsilon} e^{1-x}+A e^{-x / \varepsilon}-B$,

where

$A=\frac{(1-\varepsilon) Y\left(\zeta_{p}\right)-2 e^{1-x_{p}}+2 \varepsilon}{(1-\varepsilon)\left[e^{-x_{p} / \varepsilon}-e^{-1 / \varepsilon}\right]}$,

$B=\frac{2 \varepsilon}{1-\varepsilon}+A e^{-1 / \varepsilon}$.

In Table 2, we compare the ME1 of $\left|u_{e}-u\right|$, the ME2 of $\left|u_{e}-u_{a}\right|$, and the ME3 of $\left|u_{e}-Y\right|$ in the inner region and $\left|u_{e}-y\right|$ in the outer region for different values of $\varepsilon$, with $\zeta_{p}=1$. Composite solutions (24) and (25) are poor in the outer region, although a slightly more accurate solution is obtained in the inner region when $\varepsilon \leq 0.01$. Moreover, the composite solutions derived by Andargie and Reddy [18] are too complex to be extended to the nonlinear SPP.

The proposed new asymptotic solution (19) not only preserves the same asymptotic behavior as that of the asymptotic solution (14) but also enhances the accuracy of the entire domain. The present method is easier to use than the asymptotic matching method and is suitable for the SPP with a moderate value of the perturbing parameter. Compared with the method proposed by Andargie and Reddy [18], the present method is easier to follow and can be extended to quasilinear SPP directly.

\section{Decomposition to inner and outer problems}

We now consider a second-order quasilinear boundary value problem under the Dirichlet boundary conditions:

$\varepsilon u^{\prime \prime}(x)+p(x, u) u^{\prime}(x)+q(x, u)=0,0<x<1$,

$u(0)=\alpha, u(1)=\beta$.

Suppose that $p, q \in C[0,1]$. When $0<\varepsilon \ll 1$, an SPP is encountered. The exhibition of boundary

Table 2. Comparing ME1 (I), ME2 (I), ME3 (I), ME1 (O), ME2 (O), and ME3 (O) with different $\varepsilon$ at inner region and outer region

\begin{tabular}{lllll}
\hline$\varepsilon$ & 0.05 & 0.01 & 0.001 & 0.0001 \\
\hline ME1 (I) & $7.186 \times 10^{-2}$ & $1.736 \times 10^{-2}$ & $1.798 \times 10^{-3}$ & $1.804 \times 10^{-4}$ \\
ME2 (I) & $7.186 \times 10^{-2}$ & $1.736 \times 10^{-2}$ & $1.798 \times 10^{-3}$ & $1.804 \times 10^{-4}$ \\
ME3 (I) & $9.506 \times 10^{-2}$ & $1.681 \times 10^{-2}$ & $1.637 \times 10^{-3}$ & $1.633 \times 10^{-4}$ \\
ME1 (O) & $3.988 \times 10^{-2}$ & $7.470 \times 10^{-3}$ & $7.369 \times 10^{-4}$ & $7.359 \times 10^{-5}$ \\
ME2 (O) & $3.988 \times 10^{-2}$ & $7.470 \times 10^{-3}$ & $7.369 \times 10^{-4}$ & $7.359 \times 10^{-5}$ \\
ME3 (O) & $1.054 \times 10^{-1}$ & $2.020 \times 10^{-2}$ & $2.002 \times 10^{-3}$ & $2.000 \times 10^{-4}$ \\
\hline
\end{tabular}

layers at one or both ends of the interval depends on the properties of $p$. Under the assumption $p>0$, the boundary layer occurs near the left-end point.

Motivated by the analysis conducted in Section 2, we make the following approximation for the SPP:

$p\left(x, u_{o}\right) u_{o}^{\prime}(x)+q\left(x, u_{o}\right)=0, u_{o}(1)=\beta$,

$\varepsilon u_{i}^{\prime \prime}(x)+p(x, u) u_{i}^{\prime}(x)=0, u_{i}(0)=\alpha-u_{o}(0), u_{i}(1)=0$,

where $u(x)=u_{o}(x)+u_{i}(x)$. We neglect $u_{o}^{\prime}(x)$ in Eq. (31), which, when compared with $u_{i}^{\prime}(x)$, is small within the boundary layer. Eq. (31) is a novel boundary layer correction problem.

As indicated by Liu [1], Eq. (28) with $0<\varepsilon \ll 1$ is stiff within the boundary layer. To integrate differential equations (30) and (31), the following transformation between the independent variables $x$ and $t$ is considered:

$x(t)=1-\frac{\tanh [\lambda(1-t)]}{\tanh \lambda}, x(0)=0, x(1)=1$.

It follows from Eqs. (30)-(32) that

$$
\begin{aligned}
& \dot{u}_{o}(t)=F_{1}\left(t, u_{o}\right):=-\frac{\lambda e(t) q\left(t, u_{o}\right)}{p\left(t, u_{o}\right)}, u_{o}(1)=\beta, \\
& \ddot{u}_{i}(t)=F_{2}\left(t, u_{i}+u_{o}, \dot{u}_{i}\right) \\
& :=\left[2 \lambda \tanh [\lambda(1-t)]-\frac{\lambda e(t)}{\epsilon} p\left(t, u_{i}+u_{o}\right)\right] \dot{u}_{i}(t), \\
& u_{i}(0)=\alpha-u_{o}(0), u_{i}(1)=0,
\end{aligned}
$$

where

$e(t):=\frac{1-\tanh ^{2}[\lambda(1-t)]}{\tanh \lambda}$.

\section{A novel asymptotic numerical method}

In an approach that differs from that encapsulated by Eqs. (30) and (31), Wang [19] has proposed the following numerical method:

$$
p(x, v) \frac{d v(x)}{d x}+q(x, v)=0, v(1)=\beta,
$$

$\frac{d^{2} w(\zeta)}{d \zeta^{2}}+p(\mathbf{0}, v(0)+w(\zeta)) \frac{d w(\zeta)}{d \zeta}=0$,

$w(0)=\alpha-v(0), \lim _{\zeta \rightarrow \infty} w(\zeta)=0, \zeta:=\frac{x}{\varepsilon}$.

Wang [19] proved that $u_{w}(x)=v(x)+w(\zeta)+\mathrm{O}(\varepsilon)$ is an asymptotic solution to Eqs. (28) and (29). Wang's method is less accurate than that evident in Eqs. (30) 
and (31). Padmaja and Reddy [29] developed a numerical patching method with Padé approximates to solve the linear SPP according to the above idea. Eqs. (31) and (37) are different in three aspects: the coordinates $x$ and $\zeta$, the coefficients $p(x, u)$ and $p(0$, $v(0)+w(\zeta))$, and the right boundary conditions $u_{i}(1)=0$ and $\lim _{\zeta \rightarrow \infty} w(\zeta)=0$.

\subsection{Two theorems}

When $p$ and $q$ are nonlinear functions of $(x, u)$, the analytic asymptotic solution is not easy to obtain from the exact solutions of Eqs. (30) and (31). Therefore, we develop a novel numerical method to acquire the asymptotic numerical solution. Before deriving a novel iterative method to solve Eqs. (33) and (34), we derive the following results.

Theorem 1. For any free function $z(t) \in C[0,1]$, the function

$u_{o}(t)=z(t)-G_{1}(t)$

satisfies $u_{o}(1)=\beta$, where

$G_{1}(t):=e^{t-1}[z(1)-\beta]$.

Proof. It is obvious that

$$
\begin{aligned}
u_{o}(1)=z(1)-G_{1}(1) & =z(1)-e^{1-1}[z(1)-\beta] \\
& =z(1)-[z(1)-\beta]=\beta ;
\end{aligned}
$$

hence, we prove that $u_{o}(t)$ in Eq. (38) satisfies the right-end boundary condition in Eq. (33), i.e., $u_{o}(1)=\beta$.

Theorem 2. For any free function $y(t) \in C[0,1]$, the function

$u_{i}(t)=y(t)-G_{2}(t)$

satisfies the boundary conditions in Eq. (34), where

$G_{2}(t):=(1-t)\left[y(0)-\alpha+u_{o}(0)\right]+t y(1)$.

Proof. In Eqs. (40) and (41), we insert $t=0$ to obtain

$u_{i}(0)=y(0)-\left[y(0)-\alpha+u_{o}(0)\right]=\alpha-u_{o}(0)$.

In Eqs. (40) and (41), we insert $t=1$ to obtain

$u_{i}(1)=y(1)-G_{2}(1)=y(1)-y(1)=0$.

Thus, we end the Proof. $\square$

\subsection{Transforming to the IVP}

Here we demonstrate that Theorems 1 and 2 are useful in the asymptotic numerical solution of the SPP. In Theorems 1 and 2 , we let $z(t) \in C^{1}[0,1]$ and $y(t) \in C^{2}[0,1]$.

The insertion of Eq. (38) for $u_{o}(t)$ in Eq. (33) yields

$$
\begin{aligned}
\dot{z}(t)=H_{1}(t, z(t) ; z(1)):= & \dot{G}_{1}(t)+F_{1}\left(t, z(t)-G_{1}(t)\right) \\
= & e^{t-1}[z(1)-\beta] \\
& +F_{1}\left(t, z(t)-G_{1}(t)\right) .
\end{aligned}
$$

Similarly, the insertion of Eq. (40) for $u_{i}(t)$ in Eq. (34) yields

$$
\begin{aligned}
\ddot{y}(t) & =H_{2}(t, z(t), y(t), \dot{y}(t) ; z(1), y(1)) \\
& :=F_{2}\left(t, z(t)-G_{1}(t)+y(t)-G_{2}(t), \dot{y}(t)-\dot{G}_{2}\right),
\end{aligned}
$$

where

$\dot{G}_{2}:=\alpha-u_{o}(0)-y(0)+y(1)$

is a constant. As shown in Eqs. (39), (41) and (46), $G_{1}(t), \dot{G}_{1}(t), G_{2}(t)$, and $\dot{G}_{2}$ include two unknown values, $z(1)$ and $y(1)$, when $y(0)$ is given and $u_{o}(0)$ by Eq. (38) is given by

$$
u_{o}(0)=z(0)-G_{1}(0)=z(0)-e^{-1}[z(1)-\beta] .
$$

At the same time,

$\dot{G}_{2}=\alpha-z(0)+e^{-1}[z(1)-\beta]-y(0)+y(1)$.

Consequently, $H_{1}$ is a function of $z(1)$, as denoted in Eq. (44), and $\mathrm{H}_{2}$ is a function of $z(1)$ and $y(1)$, as denoted in Eq. (45).

Suppose that Eqs. (44) and (45) are subjected to zero initial values:

$z(0)=0, y(0)=0, \dot{y}(0)=0$.

In the IVP of Eqs. (44), (45) and (49), two unknown values, $y(1)$ and $z(1)$, are to be determined.

Let

$y_{1}(t):=z(t), y_{2}(t):=y(t), y_{3}(t):=\dot{y}(t)$,

and it follows from Eqs. (44) and (45) that

$\dot{y}_{1}(t)=H_{1}\left(t, y_{1}(t) ; y_{1}(1)\right), \dot{y}_{2}(t)=y_{3}(t)$,

$\dot{y}_{3}(t)=H_{2}\left(t, y_{1}(t), y_{2}(t), y_{3}(t) ; y_{1}(1), y_{2}(1)\right)$,

where $y_{1}(1)=: c$ and $y_{2}(1)=: d$ are two constants to be determined. If $y_{1}(1)$ and $y_{2}(1)$ are available, the differential equations in Eq. (51) are definite and we can apply the fourth-order Runge-Kutta method (RK4) to obtain $y_{1}(t)=z(t)$ and $y_{2}(t)=y(t)$. We can 
obtain $u(t)$ from $u(t)=u_{0}(t)+u_{i}(t)=y_{1}(t)-G_{1}(t)+$ $y_{2}(t)-G_{2}(t)$. The initial values $y_{1}(0)=y_{2}(0)=$ $y_{3}(0)=0$ will be adopted in all numerical examples given below.

Some numerical techniques for solving the second-order SPP are based on the concept of replacing this problem with IVPs. Different initial value methods exist in the literature [30-36]. Some methods consist of replacing the original SPP with an asymptotically equivalent first-order differential equation system and solving it as the initial value problem. Reddy and Chakravarthy [33] factorized the original problem in three first-order IVPs that differ from our approaches given in the preceding.

\subsection{The iterative algorithm}

To acquire the asymptotic numerical solution of $u$, the current method is (i) giving $c_{0}, d_{0}, \epsilon$, and $N$, (ii) repeating $k=0,1,2, \ldots$ until convergence, integrating

$\dot{y}_{1}(t)=H_{1}\left(t, y_{1}(t) ; c_{k}\right), \dot{y}_{2}(t)=y_{3}(t)$,

$\dot{y}_{3}(t)=H_{2}\left(t, y_{1}(t), y_{2}(t), y_{3}(t) ; c_{k}, d_{k}\right)$

by using the RK4 with $N$ steps from $t=0$ to $t=1$, and taking

$c_{k+1}=y_{1}(1), d_{k+1}=y_{2}(1)$.

If

$\sqrt{\left(c_{k+1}-c_{k}\right)^{2}+\left(d_{k+1}-d_{k}\right)^{2}}<\epsilon$

is satisfied, the iterations are terminated and $u(t)$ is given by

$$
\begin{aligned}
& u(t)=y_{1}(t)+y_{2}(t)-G_{1}(t)-G_{2}(t) \\
& =y_{1}(t)+y_{2}(t)-e^{t-1}\left[c_{k}-\beta\right]+(1-t)\left\{\alpha+e^{-1}\left[c_{k}-\beta\right]\right\} \\
& \quad-t d_{k},
\end{aligned}
$$

where $c_{k}$ and $d_{k}$ are the convergent values of the sequences $c_{k}$ and $d_{k}, k=1,2, \ldots$.

In some cases, $u_{o}(x)$ in Eq. (30) can be derived exactly; for such cases, only Eq. (31) and the last two equations in Eq. (51) are required to determine $u_{i}(x)$, and thus, only the unknown constant $d_{k}$ must be determined.

\section{Numerical examples}

For most SPPs there are no closed-form solutions. Therefore, we apply the initial value problem method (IVPM) developed in Ref. [13] to compute the solutions, which are then used as the referenced "exact" solutions.

\subsection{Example 1}

Consider a variable coefficient SPP [37]:

$\varepsilon u^{\prime \prime}(x)+\left(1-\frac{x}{2}\right) u^{\prime}(x)-\frac{1}{2} u(x)=0$,

$u(0)=0, u(1)=1$,

whose asymptotic solution is given in Ref. [9]:

$u_{a}(x)=\frac{1}{2-x}-\frac{1}{2} \exp \left(\frac{x^{2} / 4-x}{\varepsilon}\right)$.

Therefore,

$u_{a}(0)=0, u_{a}(1)=1-\frac{1}{2} \exp \left(\frac{-3}{4 \varepsilon}\right)<1$,

and Eq. (54) does not exactly satisfy the right boundary condition.

First, we establish $\lambda=1, c_{0}=0, d_{0}=0, N=$ 1000 , and $\epsilon=10^{-10}$ and apply the iterative algorithm in Section 4.3 to acquire the asymptotic numerical solution of Eq. (53) with $\varepsilon=0.4$, which converges within 19 iterations, as shown in Fig. 2(a). In Fig. 2(b), we compare the asymptotic numerical solution to the asymptotic solution in Eq. (54), and we observe that improvement is achieved by using the asymptotic numerical solution.

\subsection{Example 2}

Consider a nonlinear SPP [38]:

$\varepsilon u^{\prime \prime}(x)+e^{u(x)} u^{\prime}(x)-\frac{\pi}{2} \sin \left(\frac{\pi x}{2}\right) e^{2 u(x)}=0,0<x<1$,

$u(0)=u(1)=0$.

We write the uniform approximation provided by O'Malley [38]:

$u_{a}(x)=-\ln \left(\cos \frac{\pi x}{2}+1\right)+\ln \frac{2}{2-\exp (-x /(2 \varepsilon))}$.

With $\varepsilon=0.5, \lambda=0.5, c_{0}=0, d_{0}=0, N=1000$, and $\epsilon=10^{-10}$, the iterative algorithm converges within 13 iterations, as shown in Fig. 3(a). In Fig. 3(b), we compare the asymptotic numerical solution to the solution in Eq. (57) and the IVPM solution, and we observe that improvement is achieved by using the asymptotic numerical solution.

In Table 3, we compare ME1 $:=\max \left|u_{e}-u\right|$ and $\operatorname{ME2}:=\max \left|u_{e}-u_{a}\right|$ for different values of $\varepsilon$, where $u$ 

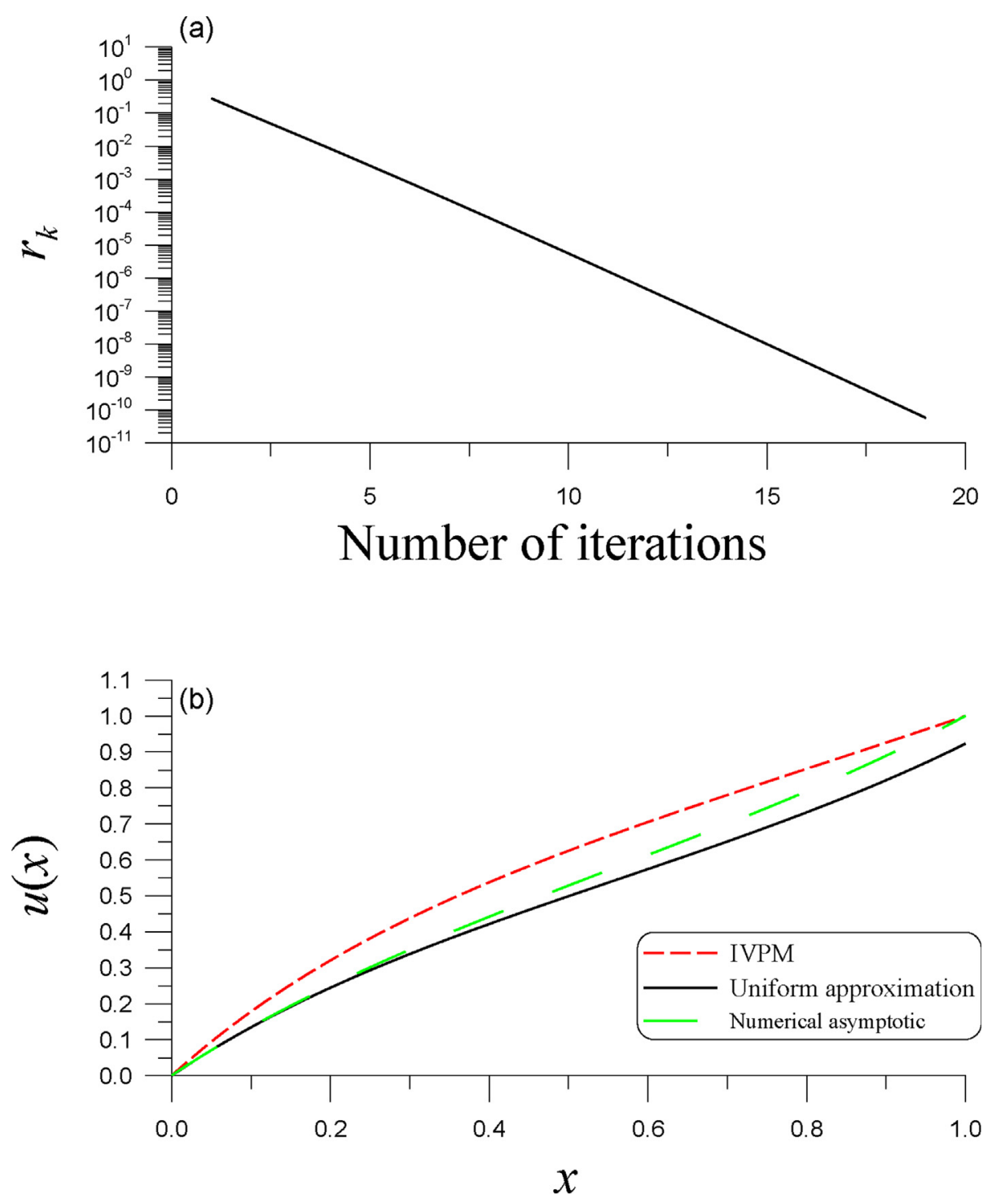

Fig. 2. Example 1 (a) demonstrates the convergence of iterations and (b) compares numerical solutions and a uniform approximation.

is the present solution, $u_{e}$ is the exact solution obtained by the IVPM, and $u_{a}$ is the solution given in Eq. (57). ME1 is smaller than ME2.

\subsection{Example 3}

Let us consider the steady-state Burgers equation:

$$
\varepsilon w^{\prime \prime}(z)-w(z) w^{\prime}(z)=0,-1<z<1
$$
$w(-1)=0, w(1)=-1$.

For the purpose of comparison, we write the uniform approximation provided by O'Malley [38]:

$w_{a}(z)=-\frac{1-\exp [-(1+z) / \varepsilon]}{1+\exp [-(1+z) / \varepsilon]}$.
Upon letting $x=(1+z) / 2$, we have

$\varepsilon w^{\prime \prime}(x)-2 w(x) w^{\prime}(x)=0$, $w(0)=0, w(1)=-1$.

When we have $w_{o}(x)=-1$, the governing equation for $w_{i}(x)$ is

$\varepsilon w_{i}^{\prime \prime}(x)-2\left[w_{i}(x)-1\right] w_{i}^{\prime}(x)=0$,

$w_{i}(\mathbf{0})=1, w_{i}(\mathbf{1})=0$.

With $\lambda=0.8, d_{0}=0, N=1000$, and $\epsilon=10^{-10}$, as shown in Fig. 4(a), the solution to Eq. (60) with $\varepsilon=$ 0.8 is determined by the IVPM with 22 iterations, and the current method reaches convergence with 22 iterations. Fig. 4(b) compares the IVPM solution to the uniform approximation in Eq. (59), with the 

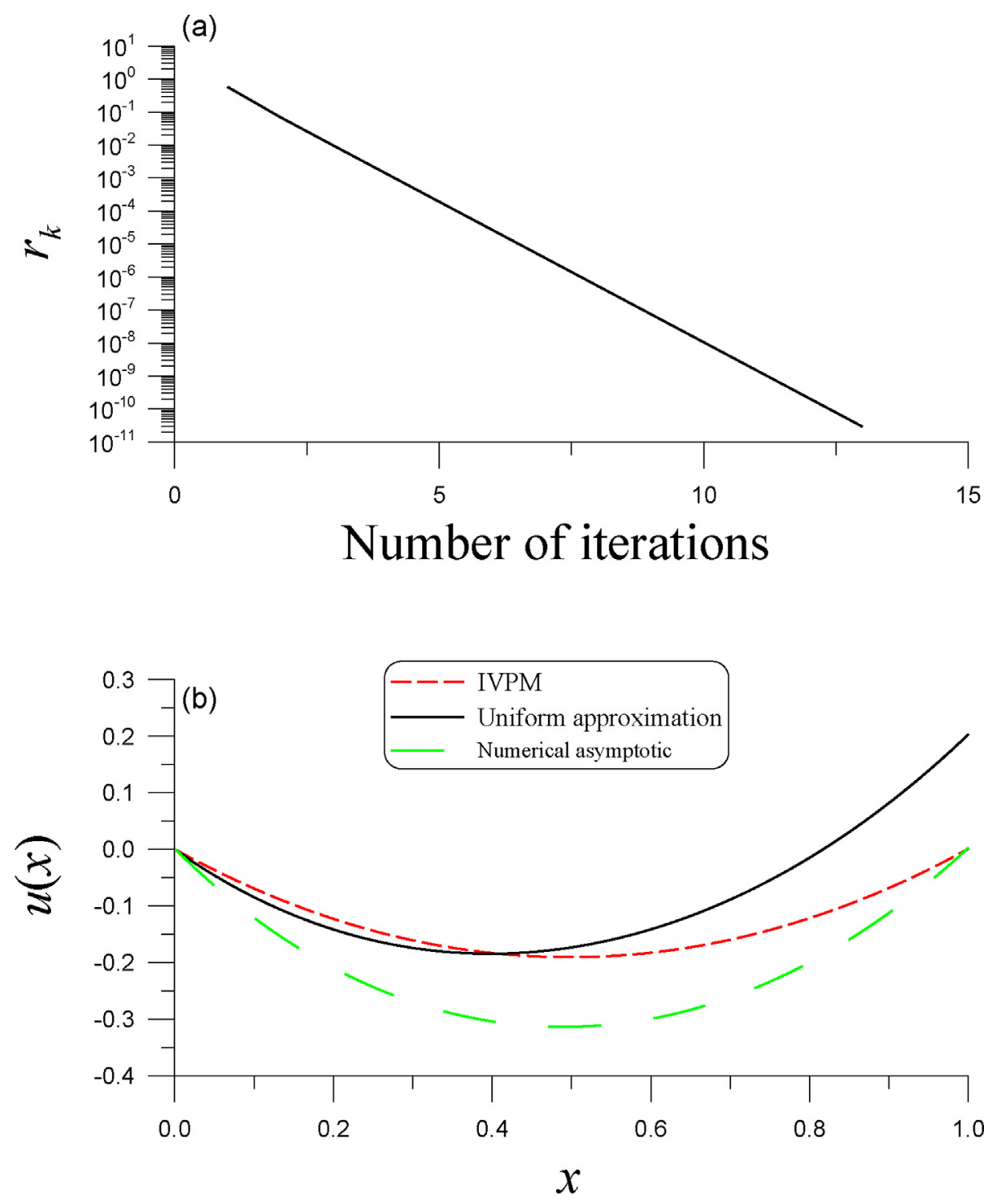

Fig. 3. Example 2 (a) demonstrates the convergence of iterations and (b) compares numerical solutions and a uniform approximation.

absolute difference being $1.524 \times 10^{-1}$ and that of the current solution being $2.22 \times 10^{-16}$. The current solution is much more accurate than the uniform approximation in Eq. (59).

Table 3. For example 2, a comparison of ME1 and ME2 with different $\varepsilon$

\begin{tabular}{lllll}
\hline$\varepsilon$ & 0.5 & 0.4 & 0.02 & 0.01 \\
\hline ME1 & $1.233 \times 10^{-1}$ & $1.161 \times 10^{-1}$ & $2.923 \times 10^{-2}$ & $8.869 \times 10^{-2}$ \\
ME2 & $2.025 \times 10^{-1}$ & $1.552 \times 10^{-1}$ & $4.744 \times 10^{-2}$ & $1.861 \times 10^{-1}$ \\
\hline
\end{tabular}

\subsection{Example 4}

Consider [36,37]:

$\varepsilon u^{\prime \prime}(x)+u(x) u^{\prime}(x)-u(x)=0$, $u(0)=-1, u(1)=3.9995$.

We write a uniform approximation provided by Kevorkian and Cole [37] as

$u_{a}(x)=x+c_{1} \tanh \left[c_{1}\left(x / \varepsilon+c_{2}\right) / 2\right]$,

where $c_{1}=2.9995$ and $c_{2}=1 / c_{1} \ln \left[\left(c_{1}-1\right) /\left(c_{1}+1\right)\right]$.

We have $u_{o}(x)=x+2.9995$, and the governing equation for $u_{i}(x)$ is 


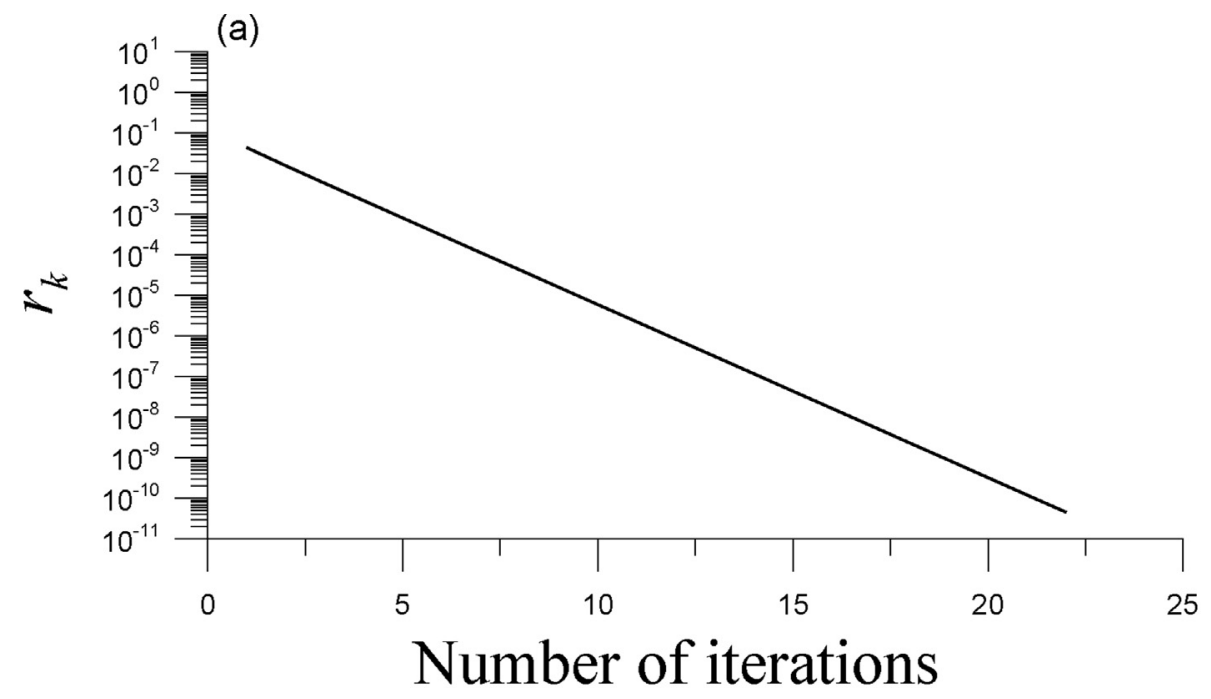

(b)

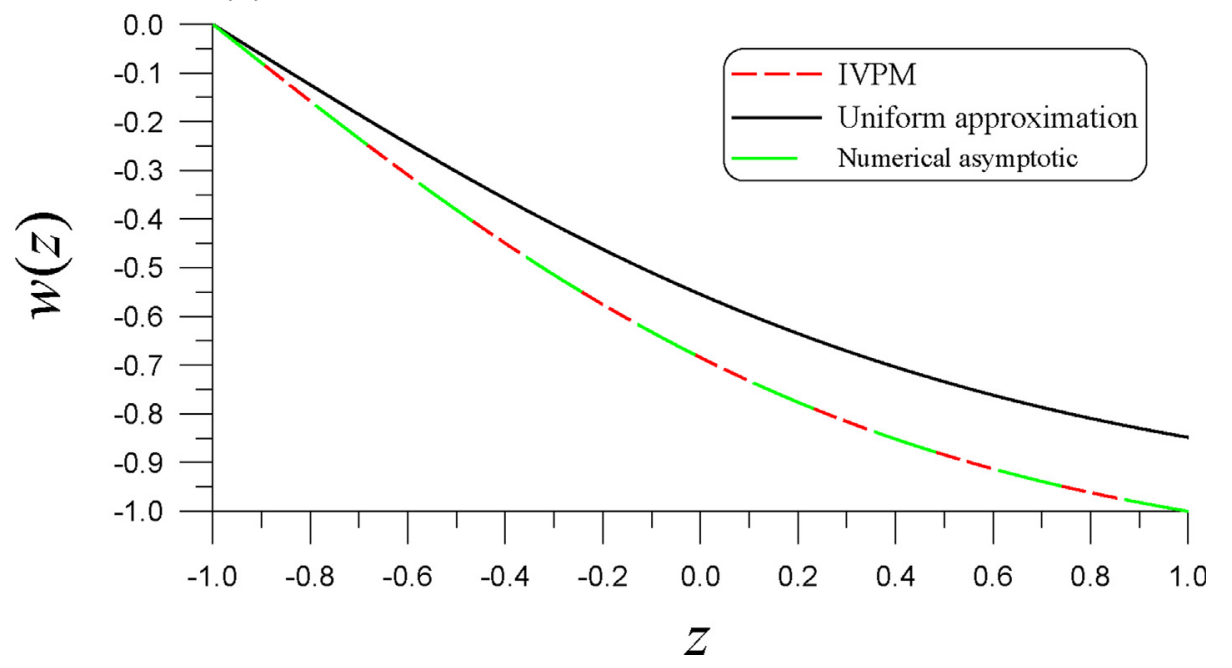

Fig. 4. Example 3 (a) demonstrates the convergence of iterations and (b) compares numerical solutions and a uniform approximation.

$\varepsilon u_{i}^{\prime \prime}(x)+\left[u_{i}(x)+u_{o}(x)\right] u_{i}^{\prime}(x)=0$,

$u_{i}(0)=-1-u_{o}(0)=-3.9995, u_{i}(1)=0$.

The current method under $\lambda=2, d_{0}=0, N=$ 1000 , and $\epsilon=10^{-10}$ is used to determine the solution of Eq. (64) with $\varepsilon=0.5$, and as shown in Fig. 5(a), it converges with 14 iterations. Fig. 5(b) compares the current solution to the uniform approximation in Eq. (63) and the IVPM solution of Eq. (62), which is deemed an exact solution. When the maximal absolute difference is 0.363 for uniform approximation and the IVPM solution, the IVPM solution with the current solution is $3.661 \times 10^{-11}$. With a large $\varepsilon=$ 0.5 , the current solution is considerably more accurate than the uniform approximation in Eq. (63).
When Wang's method [19] in Eqs. (36) and (37) is applied to solve this problem, we determine that the maximum absolute difference is 0.323 , which is slightly more accurate than the uniform approximation in Eq. (63). As shown in Fig. 5(b), Wang's solution is close to the uniform approximation. In Table 4, we compare ME1 $:=\max \left|u_{e}-u\right|$, ME2 $:=$ $\max \left|u_{e}-u_{a}\right|$, and ME3 $:=\max \left|u_{e}-u_{w}\right|$ for different values of $\varepsilon$, where $u$ is the present solution, $u_{e}$ is the exact solution obtained by the IVPM, and $u_{w}$ is the solution obtained by Wang's method. ME2 and ME3 are the same when $\varepsilon \leq 0.1$. In all ranges of $\varepsilon \leq 0.5$, ME1 is considerably smaller than ME2 and ME3. 

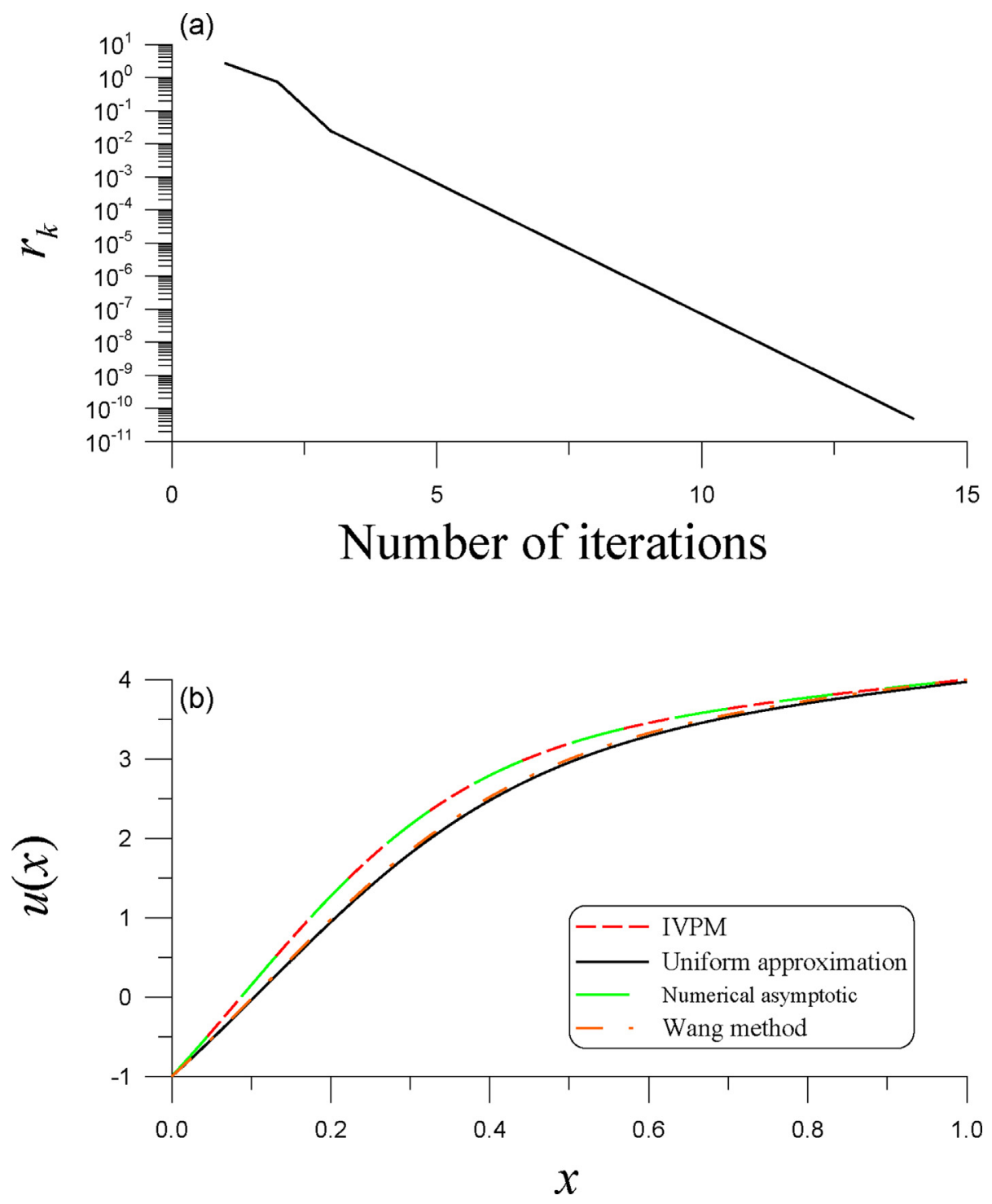

Fig. 5. Example 4 (a) demonstrates the convergence of iterations and (b) compares numerical solutions and a uniform approximation.

\subsection{Example 5}

\section{Consider [39]:}

$\varepsilon u^{\prime \prime}(x)+2 u^{\prime}(x)+e^{u(x)}=0$, $u(0)=0, u(1)=0$.

The boundary layer analysis is applied to this example, whose uniform approximation is [36]

Table 4. Comparing ME1, ME2, and ME3 of the present and asymptotic solutions to the exact solution with different $\varepsilon$

\begin{tabular}{llll}
\hline$\varepsilon$ & ME1 & ME2 & ME3 \\
\hline 0.5 & $3.661 \times 10^{-11}$ & 0.363 & 0.323 \\
0.1 & $4.718 \times 10^{-11}$ & $8.733 \times 10^{-2}$ & $8.733 \times 10^{-2}$ \\
0.05 & $1.059 \times 10^{-10}$ & $4.512 \times 10^{-1}$ & $4.512 \times 10^{-1}$ \\
0.01 & $1.798 \times 10^{-9}$ & $9.278 \times 10^{-3}$ & $9.278 \times 10^{-3}$ \\
\hline
\end{tabular}

$$
u_{a}(x)=\ln \frac{2}{x+1}-\ln 2 e^{-2 x / \varepsilon} \text {. }
$$

It does not match the boundary conditions:

$u_{a}(0)=0, u_{a}(1)=-e^{-2 / \varepsilon} \ln 2 \neq 0$.

Therefore, we have

$$
u_{o}(x)=\ln \frac{2}{x+1},
$$

and the governing equation for $u_{i}(x)$ is

$\varepsilon u_{i}^{\prime \prime}(x)+2 u_{i}^{\prime}(x)=0$,

$u_{i}(0)=-u_{o}(0)=-\ln 2, u_{i}(1)=0$,

which has a closed-form solution given by 

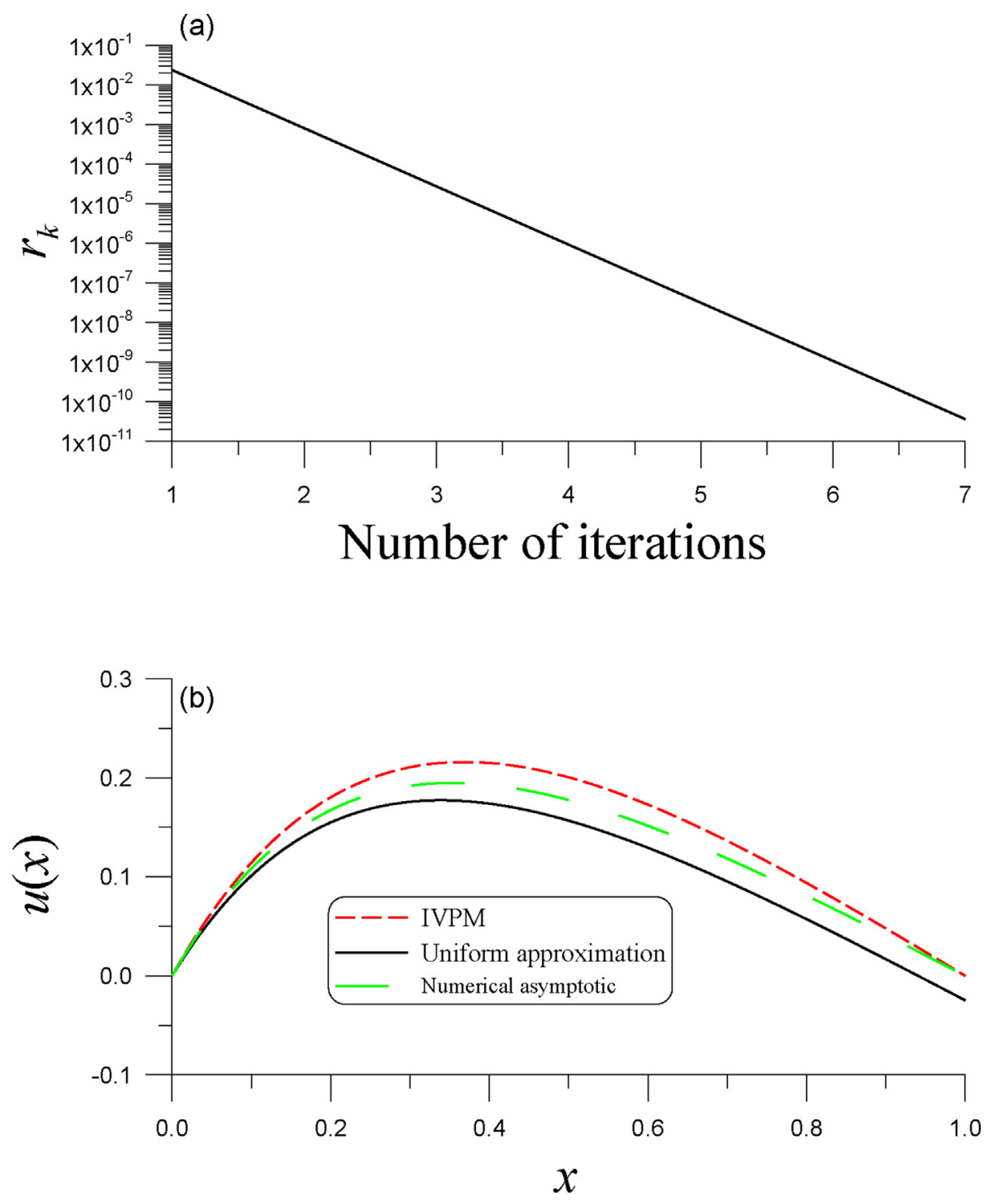

Fig. 6. Example 5 (a) demonstrates the convergence of iterations and (b) compares numerical solutions and a uniform approximation.

$u(x)=\ln \frac{2}{x+1}+\frac{e^{-2 / \varepsilon} \ln 2}{1-e^{-2 / \varepsilon}}-\frac{\ln 2}{1-e^{-2 / \varepsilon}} e^{-2 x / \varepsilon}$.

It is a new asymptotic solution, which satisfies the boundary conditions with

$u(0)=0, u(1)=0$.

Since

$e^{-2 / \varepsilon} \rightarrow 0$ when $\varepsilon \rightarrow 0$

$u(x)$ in Eq. (70) tends to $u_{a}(x)$ in Eq. (66), when $\varepsilon \rightarrow 0$.

The current method under $\lambda=1.5, d_{0}=0, N=$ 1000 , and $\epsilon=10^{-10}$ is used to acquire the solution of Eq. (69) with $\varepsilon=0.6$, and as shown in Fig. 6(a), it converges with seven iterations. Fig. 6(b) compares the solution with the uniform approximation in Eq. (66) and the IVPM solution of Eq. (65). When the absolute difference is $4.4 \times 10^{-2}$ for the uniform approximation $u_{a}$ and the IVPM solution, the IVPM solution with the current solution is $2.3 \times 10^{-2}$. With a large $\varepsilon=0.6$, the current solution is more accurate than the uniform approximation in Eq. (66).

\subsection{Example 6}

Example 4 is modified to

$\varepsilon u^{\prime \prime}(x)+\left(x+a_{0}\right) u(x) u^{\prime}(x)-u(x)=0$, $u(0)=-1, u(1)=3.9995$,

where $a_{0}$ is a constant. We can derive the outer solution as follows: 

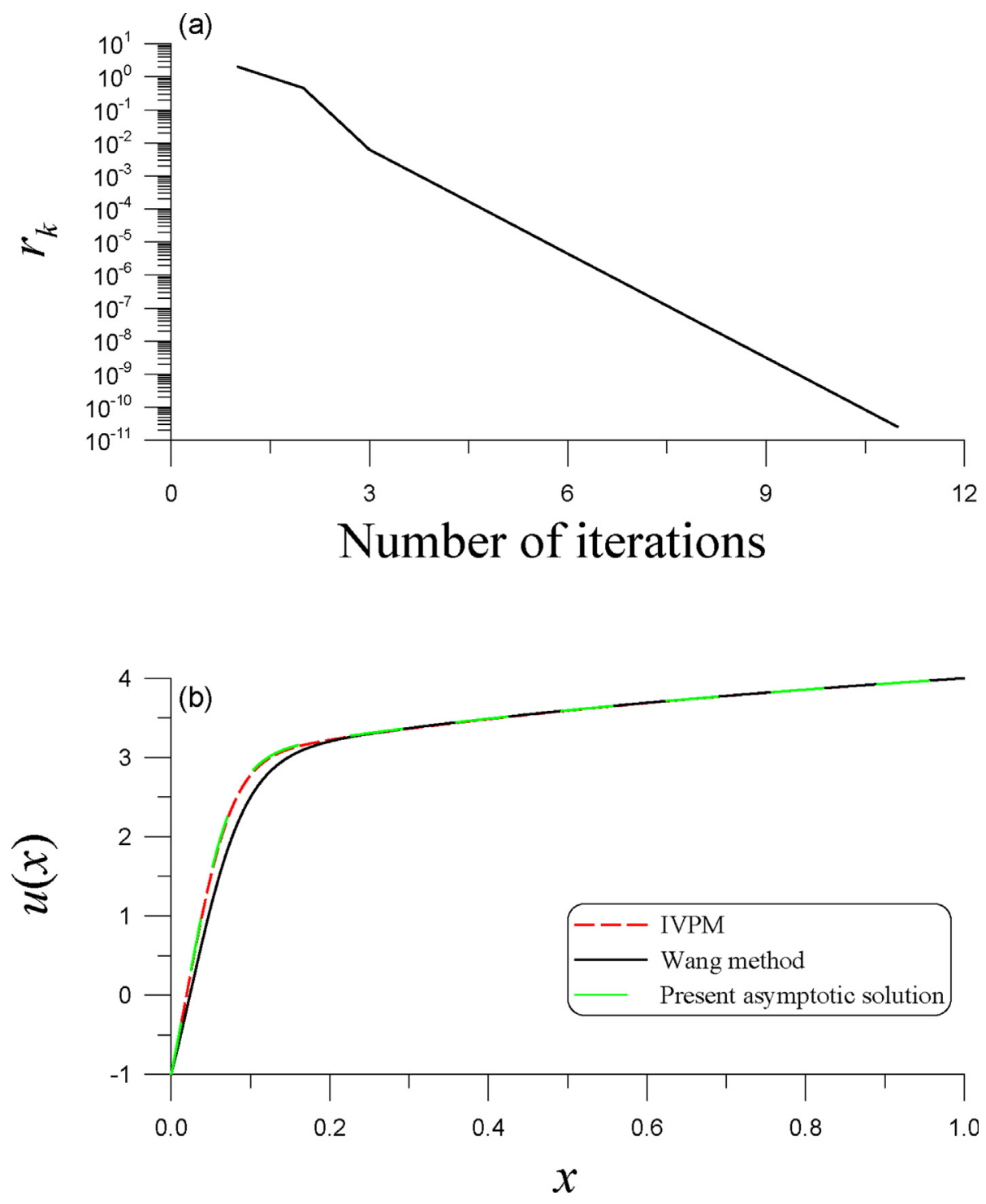

Fig. 7. Example 6 (a) demonstrates the convergence of iterations and (b) compares numerical solutions and the solution obtained from Wang's method.

$u_{o}(x)=3.9995+\ln \frac{x+a_{0}}{1+a_{0}}$

and the governing equation for $u_{i}(x)$ is

$\varepsilon u_{i}^{\prime \prime}(x)+\left(x+a_{0}\right)\left[u_{i}(x)+u_{o}(x)\right] u_{i}^{\prime}(x)=0$,

$u_{i}(0)=-1-u_{o}(0)=-4.9995-\ln \frac{a_{0}}{1+a_{0}}, u_{i}(1)=0$.

The current method under $a_{0}=0.5, \varepsilon=0.05$, $\lambda=2, d_{0}=0, N=1000$, and $\epsilon=10^{-10}$ is used to determine the solution of Eq. (74), and as shown in Fig. 7(a), it converges with 11 iterations. Fig. 7(b) compares the current solution to the IVPM solution of Eq. (72), which is deemed an exact solution.
When Wang's method [19] in Eqs. (36) and (37) is applied to solve this problem, we obtain

$\left(x+a_{0}\right) v(x) \frac{d v(x)}{d x}-v(x)=0, v(1)=3.9995$,

$\frac{d^{2} w(\zeta)}{d \zeta^{2}}+a_{0}[v(0)+w(\zeta)] \frac{d w(\zeta)}{d \zeta}=0$

$w(0)=-1-v(0), \lim _{\zeta \rightarrow \infty} w(\zeta)=0, \zeta:=\frac{x}{\varepsilon}$

where $v(x)$ is the outer solution given in Eq. (73). When $a_{0} \rightarrow 0$, Wang's method fails, owing to

$\frac{d^{2} w(\zeta)}{d \zeta^{2}}=0, w(0)=-1-v(0), \lim _{\zeta \rightarrow \infty} w(\zeta)=0, \zeta:=\frac{x}{\varepsilon}$, 
which leads to an incorrect inner solution.

As shown in Fig. 7(b), Wang's solution to Eqs. (75) and (76) deviates from the IVPM solution of Eq. (72), with a maximum absolute difference of 0.399 and a maximum absolute difference of $3.06 \times 10^{-2}$. for the current asymptotic solution.

\section{Conclusions}

Because of the existence of a boundary layer for the second-order SPP, an asymptotic numerical solution that can exactly match the boundary conditions is essential. For the SPP, we have proposed a novel boundary layer correction problem that can accurately capture the main asymptotic behavior within the boundary layer and simultaneously preserve the given Dirichlet boundary conditions. Therefore, the new asymptotic solution is an improvement upon the conventional asymptotic solution. Resorting to the functions derived in Theorems 1 and 2, we have exactly transformed the quasilinear SPP to the IVPs for two new variables with zero initial conditions. A newly developed iterative algorithm thus converges to promptly determine the two unknown right-end values of the new variables and the singularly perturbed asymptotic solution. Based on this new concept, we provide a modification to the conventional asymptotic solution of the SPP such that the asymptotic numerical solution exactly satisfies the boundary conditions. We address the accuracy of the asymptotic numerical solution, and the applicable range of the perturbing parameter in the modified asymptotic solution can be extended to a moderate value. More essentially, the modified asymptotic solution possesses the same asymptotic behavior as the conventional asymptotic solution.

\section{References}

[1] Liu CS. The Lie-group shooting method for singularly perturbed two-point boundary value problems. Comput Model Eng Sci 2006;15:179-96.

[2] Awoke A, Reddy YN. An exponentially fitted special second-order finite difference method for solving singular perturbation problems. Appl Math Comput 2007;190: 1767-82.

[3] Patidar KC. High order parameter uniform numerical method for singular perturbation problems. Appl Math Comput 2007;188:720-33.

[4] Vigo-Aguiar J, Natesan S. An efficient numerical method for singular perturbation problems. J Comput Appl Math 2006; 192:132-41.

[5] Lin TC, Schultza DH, Zhang W. Numerical solutions of linear and nonlinear singular perturbation problems. Comput Math Appl 2008;55:2574-92.

[6] Liu CS. The Lie-group shooting method for solving nonlinear singularly perturbed boundary value problems. Commun Nonlinear Sci Numer Simulat 2012;17:1506-21.
[7] Khuri SA, Sayfy A. Self-adjoint singularly perturbed boundary value problems: an adaptive variational approach. Math Methods Appl Sci 2013;36:1070-9.

[8] Dogan N, Erturk VS, Akin O. Numerical treatment of singularly perturbed two-point boundary value problems by using differential transformation method. Discrete Dynam Nat Soc 2012. Article ID 579431:10 pages.

[9] El-Zahar ER. Approximate analytical solution for singularly perturbed boundary value problems by multi-step differential transform method. J Appl Sci 2012;12:2026-34.

[10] Liu CS. Solving singularly perturbed problems by a weakform integral equation with exponential trial functions. Appl Math Comput 2018;329:154-74.

[11] Liu CS, Chang CW. Solving nonlinear singularly perturbed problems by fractional order exponential trial functions. Appl Math Lett 2018;83:219-26.

[12] Liu CS, Li B. Solving second-order singularly perturbed ODE by the collocation method based on energetic Robin boundary functions. Appl Math 2019;64:679-93.

[13] Liu CS, Chang JR. Boundary shape functions methods for solving the nonlinear singularly perturbed problems with Robin boundary conditions. Int J Nonlinear Sci Numer Stimul 2020;21:797-806.

[14] Roos HG, Stynes M, Tobiska L. Numerical methods for singularly perturbed differential equations. Berlin: SpringerVerlag; 1996.

[15] Awoke A, Reddy YN. Terminal boundary condition for singularly perturbed two-point boundary value problems. Neural, Parallel Sci Comput 2008;16:435-48.

[16] Chakravarthy PP, Reddy YN. A cutting point technique for singular perturbation problems. J Math Contr Sci Appl 2007; 1:39-59.

[17] Vigo-aguiar J, Natesan S. A parallel boundary value technique for singularly perturbed two-point boundary value problems. J Supercomput 2004;27:195-206.

[18] Andargie A, Reddy YN. The method of asymptotic inner boundary condition for singular perturbation problems. J Appl Math Inform 2011;29:937-48.

[19] Wang L. A novel method for a class of nonlinear singular perturbation problems. Appl Math Comput 2004;156:847-56.

[20] Valanarasu T, Ramanujam N. Asymptotic initial-value method for singularly-perturbed boundary problems for second-order ordinary differential equations. J Optim Theor Appl 2003;116:167-82.

[21] Kaushik A, Kumar V, Vashishth AK. An efficient mixed asymptotic-numerical scheme for singularly perturbed convection diffusion problems. Appl Math Comput 2012;218: 8645-58.

[22] Attili BS. Numerical treatment of singularly perturbed two point boundary value problems exhibiting boundary layers. Commun Nonlinear Sci Numer Simulat 2011;16:3504-11.

[23] Liu CS, Chang JR. The periods and periodic solutions of nonlinear jerk equations solved by an iterative algorithm based on a shape function method. Appl Math Lett 2020;102. Article number 106151.

[24] Lin J, Zhang Y, Liu CS. Solving nonlinear third-order threepoint boundary value problems by boundary shape functions methods. Adv Difference Eqs 2021;2021. Article number 146.

[25] Liu CS, Chang CW. Boundary shape function method for nonlinear BVP, automatically satisfying prescribed multipoint boundary conditions. Bound Value Probl 2020;2020. Article number 139.

[26] Liu CS, Chang JR. Boundary shape functions methods for solving the nonlinear singularly perturbed problems with Robin boundary conditions. Int J Nonlinear Sci Numer Stimul 2020;21:797-806.

[27] Liu CS. A boundary shape function method for analyzing nonlinear composite beams, subjecting to nonlinear boundary moment conditions. Compos Struct 2021;262. Article number 113636.

[28] Nayfeh AH. Introduction to perturbation techniques. New York: Wiley; 1981. 
[29] Padmaja P, Reddy YN. A Numerical patching method for solving singular perturbation problems via Padé approximates. Int J Appl Sci Eng 2013;11:51-67.

[30] Gasparo MG, Maconi M. New initial value method for singularly perturbed boundary value problems. J Optim Theor Appl 1989;63:213-24.

[31] Gasparo MG, Maconi M. Initial value methods for second order singularly perturbed boundary value problems. J Optim Theor Appl 1990;66:197-210.

[32] Gasparo MG, Maconi M. Numerical solution of second-order nonlinear singularly perturbed boundary value problems by initial value methods. J Optim Theor Appl 1992;73:309-27.

[33] Reddy YN, Chakravarthy PP. An initial-value approach for solving singularly perturbed two-point boundary value problems. Appl Math Comput 2004;155:95-110.

[34] Kanshik A, Kumar V, Vashishth AK. An efficient mixed asymptotic-numerical scheme for singularly perturbed convection diffusion problems. Appl Math Comput 2012;218: 8645-58.

[35] El-Zahar ER, EL-Kabeir SMM. A new method for solving singularly perturbed boundary value problems. Appl Math Inform Sci 2013;7:927-38.

[36] El-Zahar ER. Approximate analytical solution of singularly perturbed boundary value problems in MAPLE. AIMS Math 2020;5:2272-84.

[37] Kevorkian J, Cole JD. Perturbation methods in applied mathematics. New York: Springer Science \& Business Media; 2013.

[38] O'Malley RE. Singular perturbation methods for ordinary differential equations. New York: Springer; 1991.

[39] Bender CM, Orszag SA. Advanced mathematical methods for scientists and engineers I. New York: Springer Science Business Media; 1999. 